\title{
Research Status and Prospect of Remote Intelligent Monitoring System for Engineering Blasting Vibrarion
}

\author{
Ming-shou Zhong ${ }^{1, a}$, Quan-min $\mathrm{Xie}^{2, \mathrm{~b}}$, Ying $\mathrm{Liu}^{1, \mathrm{c}}$, Jian-yu $\mathrm{Wu}^{1, \mathrm{~d}}$, Hao-quan \\ Liu $^{1, e}$ \\ ${ }^{1}$ University of Science \& Technology of PLA, Nanjing 210007, China; \\ ${ }^{2}$ Wuhan Ornance N.C.O. Academy of PLA, Wuhan 430075, China. \\ azhongms7@126.com, ${ }^{b} x i e q u a n m i n 1983 @ 163 . c o m,{ }^{c}$ Liuying@126.com, dwujianyu@163.com \\ ètao-quan@sina.com
}

Keywords: Digital blasting, Blasting vibration, Digital blasting vibration measurement.

\begin{abstract}
With the development of digital blasting, research work of digital blasting vibration measurement in recent years has become a hotspot in the field of engineering blasting. Research progress in hazard remote monitoring technology and platform of domestic engineering vibration of blasting vibration were summarized. On the basis of the analysis of the current situation of digital blasting vibration measurement, reasonable suggestions for the research of remote intelligent monitoring system for blasting vibration were proposed. The research results have reference value for promoting the development of digital blasting.
\end{abstract}

\section{Introduction}

With the rapid development of computer, internet, communication and sensor technology in recent years, modern test system has been gradually developed into the core of computer and information processing technology. Therefore, in the current hot research field of digital blasting, the blasting safety and information technology research were effectively integrated, and the remote monitoring system of blasting vibration damage effect can be made possible. Many researchers in blasting vibration monitoring and intelligent data analysis have made many meaningful explorations, and proposed key technical problems and solving measures in remote vibration process. Taking into remote vibration related to computer, communication, digital signal analysis, sensor technology and multi-disciplinary cross fusion, in recent years it has achieved some research results.

\section{Research status}

\section{Wireless monitoring system for blasting vibration based on Internet of things by China Yangtze River Science Research Institute}

The wireless blasting vibration monitoring system, based on Internet of things and wireless network technology, was developed in China Yangtze River Science Research Institute. The system is mainly composed of a detector, a blasting recorder, a central server and a terminal monitoring and management system. Among them, the blasting recorder includes wireless transmission, radio frequency identification, global positioning system function, the detector can be sampled to obtain the multi-channel signal parallel acquisition and data analysis. When the engineering blasting vibration test, signals will be converted into electric signal and transmitted through data lines to the blasting recorder. Measured datas can transmite to the central server through wireless module of blasting recorder, and can be unified management and storage. User login terminal monitoring management system through the internet, and can get the blasting vibration data [1]. 


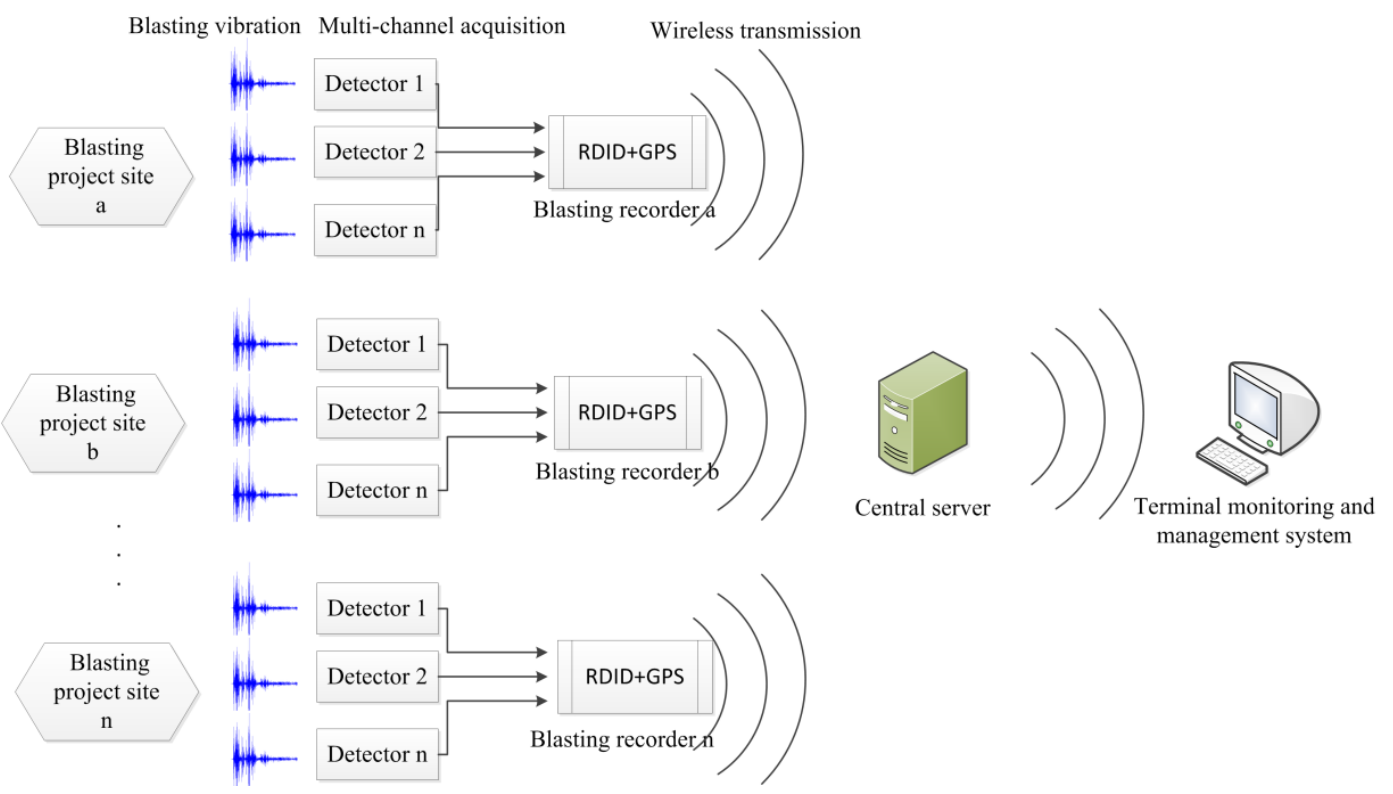

Fig.1 Schematic diagram of wireless monitoring system

\section{Blasting vibration remote monitoring system by Railway Science Institute}

China Academy of Railway Science has developed a set of blasting vibration monitoring system based on remote TC-4850N wireless network vibrometer. The system is composed of a speed sensor, TC-4850N wireless network vibrometer and special data servers. The system can be used to upload the collected data to the dedicated server through the built-in 3G network equipment.For underground or tunnel and other places,where without mobile phone signal, meared signals will be firstly uploaded by Wi-Fi module to the place where 3G signal coverage, and then through the dedicated VPN channel transmission, which can ensure the safety of data[2].

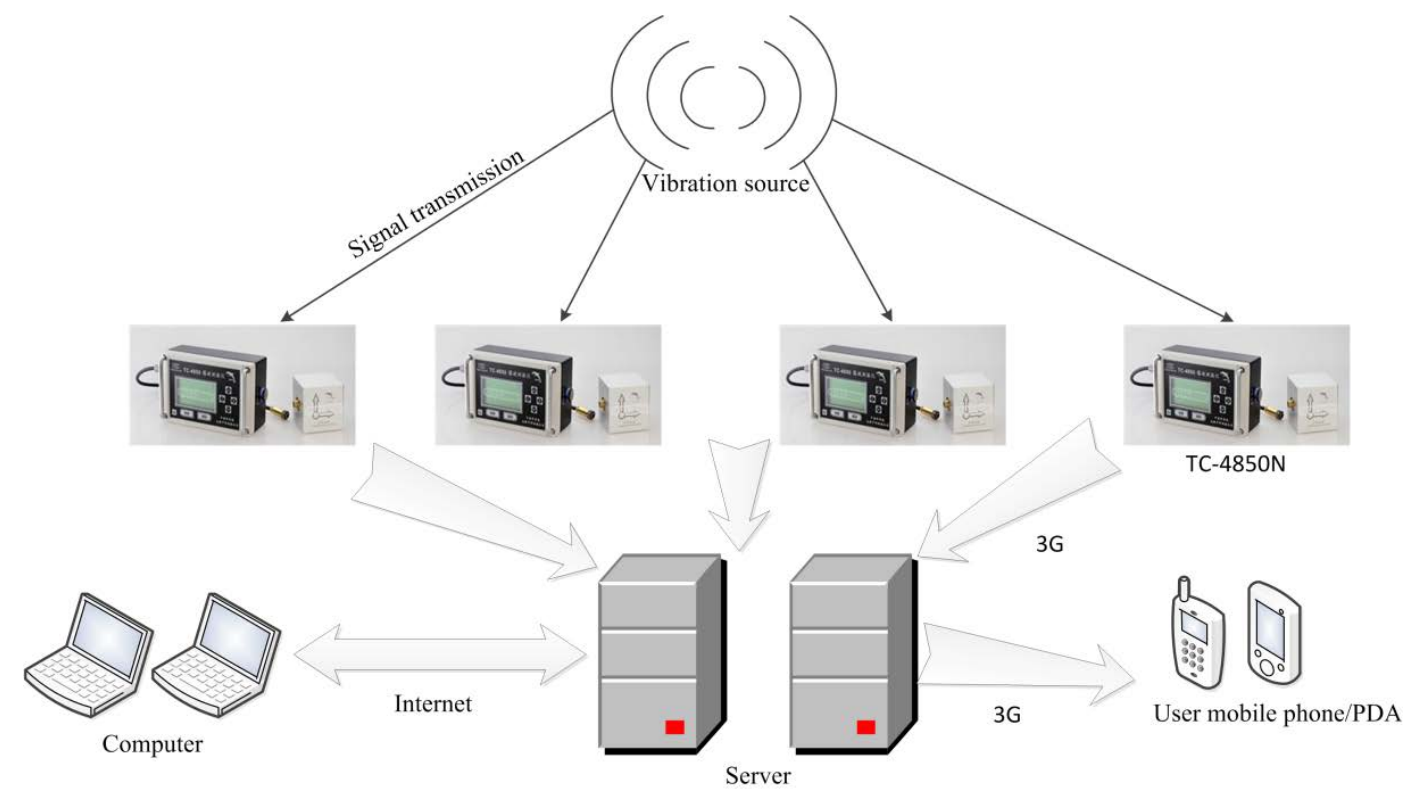

Fig.2 Schematic diagram of remote monitoring system for blasting vibration

\section{Blasting vibration prediction and control platform developed by PLA University of Science and Technology}

A blasting vibration prediction and control platform was developed by PLA University of Science and Technology. The platform contains engineering information collection, important degree analysis, forecasting model function, signal analysis, modal analysis and other five modules for blasting vibration safety construction broken control in military engineering, traffic, water conservancy, nuclear power, mine and the sea silt engineerings.

The platform uses a variety of new algorithms in digital signal analysis field. For example, the grey correlation method and rough set theory were used in the important degree analysis module to 
analyze the importance of main blasting parameters to blasting vibration parameters; The module of prediction model by the Sadauskas formula fitting algorithm and support vector machine based on the improved algorithm, to realize the prediction of blasting vibration intensity, frequency and duration; signal analysis module used the HHT, the two generation of lifting wavelet, FFT to achieve a comprehensive analysis of the time-frequency characteristics of blasting vibration test signals. The platform introduced in a variety of digital signal analysis algorithms, which can help to extract information in blasting vibration signals more accurately, and provide the core technology support for intelligent monitoring system for blasting vibration.

\section{Engineering blasting vibration remote vibration measuring system developed by Guangzhou China-Blast Digital Information Technology Co.Ltd}

Guangzhou China-Blast Digital Information Technology Co.Ltd has developed the engineering blasting vibration remote vibration measuring system based on China blasting safety web.The system relies on China blasting safety web, which composed by China Engineering Blasting cloud computing center, engineering blasting remote vibration center, engineering blasting vibration test center and network vibrometer, blasting engineering remote measuring software, blasting network in China, mobile china blasting network. Remote measuring system can provide rich data analysis function for enterprise users and vibration expert users, wich use the classical Fourier algorithm to analyse the amplitude frequency characteristics, power spectrum and time frequency energy feature for the vibration datas.

\section{Discussion and prospects}

At the beginning of 2009, China Engineering Blasting Association proposed digital blasting development philosophy in blasting engineering industry Twelfth Five Year development plan, which put forward new requirements of digital vibration for blasting vibration research. In blasting safety procedures(GB6722-2014) also clearly put forward that the D level blasting engineering blasting vibration hazards must be tested, and the test results need to enter the Chinese blasting network information system for a long time to save. Therefore, many new key technical problems based on the digital and information technology in engineering blasting vibration measurement and data analysis processing will be faced.

So far, the engineering blasting industry has not yet established technical specifications applicable for blasting vibration test work. Selection of different units of vibration system may be different, the sensors are not the same, the test data treatment methods also different, resulting vibration report content and format are also varied. In order to improve the current situation, several representative units have carried out research and development of intelligent and remote monitoring system of engineering blasting vibration, and research results have been gradually entered into popularization and application stage.

In conclusion, with the rapid development of sensor technology, computer technology, Internet technology and signal analysis technology related disciplines, digital blasting vibration research work in recent years has made rapid progress.

Next, the digital measurement of blasting work will move toward a more standardized, scientific, precise and convenient direction.

In order to improve the application range of remote intelligent monitoring system for blasting vibration damage, recommendations in the development process to focus on:

1) Research on new algorithm of digital signal analysis. Traditional algorithms of analysis precision and function, have been unable to meet the requirements of blasting fine and digital. Therefore, it is suggested for blasting vibration signal pre processing, signal feature extraction and data mining analysis methods will be introduced in the intelligent monitoring system, rich blasting vibration signal analysis function in signal system, and it will be helpful for blasting vibration damage effect control and providing more effective scientific basis.

2) Depth excavation of details characteristics for blasting vibration test data. Blasting vibration signal contains rich information about explosion source structure, transmission site medium characteristics, structural dynamic response and many others. So, we need in-depth study of the 
characteristics of the data mining algorithm and get more information.

\section{Acknowledgment}

Project fund: National Natural Science Foundation of China (51304218), Natural Science Foundation of Jiangsu province (BK20151449).

\section{References}

[1] Huang Yue-wen, Wu Xin-xia. Wireless network monitoring system of blasting vibration based on internet of things [J]. Engineering Blasting, 2012,18(1):67-70.

[2] Yang Nian-hua, Xue Li. Blasting vibration remote monitoring system and its application[J]. Engineering Blasting, 2012,18(1):71-74. 